\title{
Dietary behavior during pregnancy
}

\author{
ANDREEA CRISTIANA IORDACHESCU ${ }^{1}$, MONICA MIHAELA CIRSTOIU ${ }^{1,2}$, \\ CORINA-AURELIA ZUGRAVU ${ }^{1,3}$, OANA MIHAELA TEODOR ${ }^{2}$, \\ NATALIA TURCAN ${ }^{2,4}$, IONITA DUCU ${ }^{2}$ and ROXANA ELENA BOHILTEA ${ }^{1,2}$

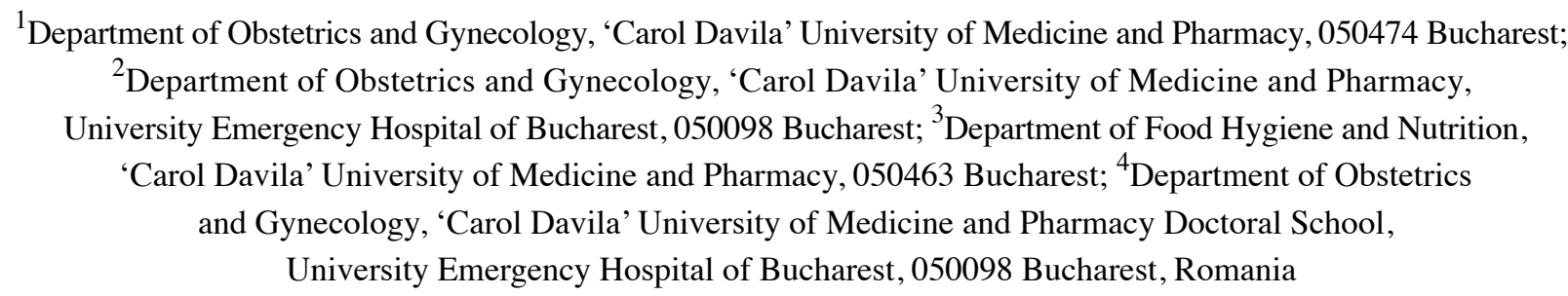

Received March 10, 2020; Accepted April 09, 2020

DOI: $10.3892 /$ etm.2020.8804

\begin{abstract}
During pregnancy, maternal diet is a modifiable factor that impacts the birth outcome. Since the nutritional needs of a pregnant women vary during preconception, gestational and breastfeeding period, it is necessary to adapt the diet and lifestyle, optimally under the personalized nutrition guidance of a specialist. High quality research regarding diet during pregnancy remains challenging as nutritional concerns also vary according to religion, financial income, age and education of the pregnant woman, as well as specific traditions of each country, limited number of dietitians with special training in maternal nutrition. The obstetrician usually plays a major role in dietary counselling and the use of nutritional monitoring tools can help identify pregnant women who may be at risk due to an inappropriate diet. The age of the pregnant women can be important when it comes to dietary and lifestyle changes. In this context, we addressed a questionnaire to 100 pregnant women in third trimester of pregnancy in order to evaluate a possible correlation between the age and the dietary behavior and lifestyle of a pregnant woman. Furthermore, the maternal compliance with dietary recommendations in pregnancy and the exposure to various risks due to unhealthy nutrition were also analysed in the study.
\end{abstract}

\section{Introduction}

Adequate maternal intake of nutrients during pregnancy supports the fetal growth and development, thus maternal

Correspondence to: Dr Natalia Turcan, Department of Obstetrics and Gynecology, 'Carol Davila' University of Medicine and Pharmacy Doctoral School, University Emergency Hospital of Bucharest, 169 Splaiul Independentei, 050098 Bucharest, Romania

E-mail: napritcan@gmail.com

Key words: dietary behavior, nutrition, pregnancy, nutrient requirements, nutrition advice malnutrition and supranutrition may have both short-term and long-term adverse fetal impact (1-3). Barker's hypothesis on the development of the diseases suggests that the fetal environment and the changes of the fetal metabolic pathways cause epigenetic changes that influence the development of diseases in childhood and adulthood (4). Therefore, it is important to evaluate, monitor and, if appropriate, take measures to optimize the maternal diet both preconceptionally and during the gestation and lactation periods.

Pregnant women should eat nutrient-dense foods to meet higher nutritional requirements during pregnancy without exceeding the caloric requirement (5). Consultation of a nutritionist with a special training in the maternal field is suitable and recommended for women with special nutritional considerations $(6,7)$ such as metabolic disorders (diabetes, hypertension, phenylketonuria, gastrointestinal disorders, bariatric surgical or digestive tract resection history with impaired absorption), modified caloric requirements [extreme age (8), multiple pregnancy, underweight, overweight, obesity (9), personal history of major weight fluctuations, another fetus with neural tube defect (10)], unhealthy eating habits (restrictive diets, eating disorders that require medication or hospitalization, intolerance and food allergies, use of toxic substances or drugs that affect nutrition). Some women might need adjusted micronutrient supplementation $(8,11,12)$.

Asking the patient to complete a questionnaire is helpful in reviewing her typical diet and in identifying obvious deficiencies (13) or potential problems such as reducing the number of meals required daily, following a special diet, the consumption of artificially sweetened beverages.

The purpose of the study was to analyse food behavior during pregnancy and to determine the extent to which pregnant women in the third trimester of pregnancy complied with the doctors' recommendations regarding nutrition and lifestyle. In the subsidiary, we aimed to verify the hypothesis that the higher the biological age of the pregnancy, the higher is the level of awareness and application of the recommendations on a healthy and balanced nutrition (based on the food pyramid). Another objective of the present research involves offering, 
based on the conclusions drawn from its development, recommendations to those involved in reducing the materno-fetal risks of poor maternal feeding and/or lifestyle.

\section{Subjects and methods}

The research data for this study was collected between 15-30.03.2018 following the application of scale instruments-questionnaire, using the pencil-paper method, on a sample of 100 pregnant women aged between 15 and 41 years in the third trimester of pregnancy. Although this version of the questionnaire has not yet been scientifically validated, a more general version has been validated to assess the risk of chronic diet-related diseases and has been successfully used in women (13).

The participants were selected by the criterion-based sampling method. Without neglecting the particular aspects, we aimed to capture the common experience of the participants, focusing on more general data. The recruitment procedure was based on convenience and a voluntary basis, the tests were applied individually, the questionnaire was completed only once, with a time limit of $\sim 20$ min to guarantee the confidentiality of the information collected. Statistical methods and tools in Microsoft Excel were used to group the results and to verify the proposed research hypothesis.

The main analyzed data concern the dietary behavior, information by the obstetrician and the extent to which pregnant women follow these recommendations, the presence of dietary restrictions, use of foods with infectious risk regarding listeriosis and toxoplasmosis, the consumption of dietary supplements, of multivitamins and minerals, lifestyle assessment (physical activity, smoking, coffee, alcohol, drugs, other medication in pregnancy).

Analysis of the correlation between pregnant biological age and dietary behavior. Starting from the hypothesis that the eating behavior is improved (body mass index-BMI is within the normal limits) as the biological age of the pregnancy is higher, the questionnaire investigated the type of food consumed the previous day and if this feeding mode is usually adhered to. Initially, the subjects had to choose from 5 food groups, presented in the questionnaire as the Food Pyramid (group 1 at the base of the pyramid includes bread, cereals, rice, pasta and potatoes, group 2 contains fruits and vegetables, group 3 includes dairy products, group 4 products such as red meat, white meat and eggs, and group 5 at the top of the pyramid contains processed sweets). In order to quantify the nutritional value of each of the five groups in relation to the other, whereas not to disadvantage a pregnant woman eating from only two groups of aliments, but of high nutritional value, compared to those who consume from all or almost all food groups, the nutritional value of food groups was ascending ranked (0-4 points) as follows: group 5 (processed sweets), 0; group 4 (white and red meat), 1; group 1 (products of pasta and cereals), 2; group 3 (dairy products), 3; and group 2 (vegetables, fruits), 4 points. In addition, to express the dietary behavior of the pregnant woman regardless of the number of food groups consumed daily, a total score was calculated as an arithmetic mean: the sum of the points related to all the groups that the pregnant women consumed the previous day divided by the number of food groups.
The final scores expressed the integrated behavior between 0 and 4 , where a score 0 corresponds to the case of eating only food in group 5 (processed sweets) and a score 4 corresponds to the case of eating only the food in group 2 (vegetables and fruits). However, even this score might not be sufficiently accurate on real food behavior of the pregnant women. For this reason, the final score should integrate a correction coefficient depending on the reccurence of the given food behavior from the previous day. To the final score was added or subtracted 0.5 points according to the following criteria: For score $<2$ from the first formula: if the pregnant woman will keep the inappropriate eating behavior, then 0.5 points are substracted; otherwise, if the pregnant woman declares that she changes the dietary behavior (presumably with a more beneficial one), then 0.5 points are added. For score $\geq 2$ (eating behavior during the previous day according to the recommendations): if the pregnant women will keep the eating behavior, then 0.5 points are added; otherwise if the food behavior is changed (supposedly with a less beneficial one) then 0.5 points are substracted. In order to achieve slightly technically this correction for the final score a Microsoft Excel IF function was used as follows: = IF $(O R(A N D(P n<2 ; Q n=' D$ ' $)$; $A N D(P n>=2$; $\left.\left.\left.Q n=' N^{\prime}\right)\right) ;-0.5 ; 0.5\right)$, where $\mathrm{Pn}$ is the final score 1 and $\mathrm{Qn}$ is the answer to question no. 2 (Yes or No). The obtained final score 2 estimates the eating behavior for each pregnant woman in the study. To the value of final score 2 was added the number of meals per day (with the mention that for the answer 'more than 3 meals' the ideal case of 5 meals per day was considered) and the values were further normalized. The next step was to verify the supposed correlation with the subject's biological age. Since there are more pregnant women of same age, there are two further levels of sorting: week of gestation and body-weight before pregnancy, resulting in 6 age categories $(15-19,20-24,25-29,30-34$, 35-39 and 40-44 years). Several parameters of interest were calculated for each age group.

Analysis of the correlation between biological age of the pregnant woman and lifestyle. To address the lifestyle assessment of pregnant women, they were questioned about regular physical activity, smoking, consumption of alcoholic beverages, coffee, medicines and recreational drugs and specific scores were assigned depending on the answer options: for the question regarding physical activity score 1 was assigned to active women and score 0 to sedentary ones; for the question regarding smoking, the score 0-3 was assigned depending on the number of cigarettes smoked per day ( 0 cigarettes/day, 3; 0 -10 cigarettes/day, 2 ; 10-20 cigarettes/day, $1 ; 20+$ cigarettes/day, 0 points); for the question regarding the consumption of coffee the score 0-2 was assigned depending on the number of cups consumed per day ( 0 cups, $2 ;<4$ cups/day, $1 ; \geq 4$ cups/day, 0 points); for the question regarding the consumption of medicines in general, respectively the consumption of medicines not prescribed by the doctor 0 points were given to the women who answered positively and 1 point for a negative answer. For each subject the points were summed up. The data was sorted by age groups, similarly to the previously used method. The aim was to verify the hypothesis that as biological age of a pregnant woman increases, the healthier her lifestyle is. 
Table I. Average values by age group within the sample of 100 pregnant women.

\begin{tabular}{|c|c|c|c|c|c|c|c|c|c|c|c|}
\hline $\begin{array}{l}\text { Age groups } \\
\text { (years) }\end{array}$ & $\mathrm{N}$ & $\begin{array}{c}\mathrm{A} \\
\text { (years) }\end{array}$ & $\begin{array}{c}\mathrm{H} \\
(\mathrm{cm})\end{array}$ & GW & $\begin{array}{l}\mathrm{W}_{1} \\
(\mathrm{~kg})\end{array}$ & $\mathrm{BMI}_{1}$ & $\begin{array}{l}\mathrm{W}_{2} \\
(\mathrm{~kg})\end{array}$ & $\mathrm{BMI}_{2}$ & $\begin{array}{l}\text { Average score } \\
\text { for DB }\end{array}$ & $\begin{array}{l}\text { Average } \\
\text { of Mpd }\end{array}$ & $X$ \\
\hline $15-19$ & 5 & 17.6 & 170.4 & 35.6 & 69.4 & 23.7 & 81.6 & 28.0 & 2.1 & 3.8 & 5.9 \\
\hline $20-24$ & 14 & 22.0 & 164.8 & 35.1 & 65.2 & 23.8 & 75.5 & 27.7 & 2.2 & 3.5 & 5.7 \\
\hline $25-29$ & 29 & 27.2 & 165.0 & 33.5 & 64.1 & 23.5 & 77.0 & 28.3 & 2.2 & 3.7 & 6.2 \\
\hline $30-34$ & 33 & 31.9 & 165.8 & 33.7 & 66.8 & 24.1 & 78.4 & 28.3 & 2.1 & 3.8 & 6.1 \\
\hline $35-39$ & 14 & 36.5 & 163.1 & 31.4 & 66.1 & 24.8 & 78.3 & 29.4 & 2.8 & 3.7 & 8.5 \\
\hline $40-44$ & 5 & 40.2 & 167.0 & 34.6 & 73.4 & 26.1 & 85.4 & 30.4 & 2.7 & 4.2 & 9.6 \\
\hline Total & 100 & 29.5 & 165.3 & 33.7 & 66.2 & 24.1 & 78.1 & 28.5 & 2.3 & 3.7 & 6.5 \\
\hline
\end{tabular}

$\mathrm{N}$, number of pregnant women in each of the six age groups, $\mathrm{A}$, average age, $\mathrm{H}$, average height, $\mathrm{GW}$, average week of gestation, $\mathrm{W}_{1}$, average weight before pregnancy, BMI, average BMI before pregnancy, $\mathrm{W}_{2}$, average weight in the third trimester, $\mathrm{BMI}_{2}$, average $\mathrm{BMI}$ in the third trimester, X, Final and standardized score for eating behavior by age groups, DB, dietary behavior, Mpd, number of meals per day.

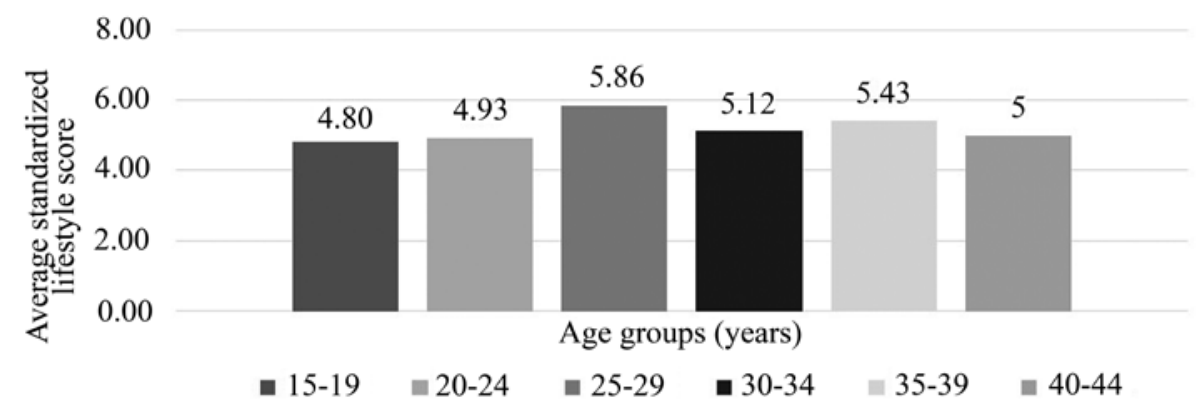

Figure 1. Average final score for the lifestyle of pregnant women by age groups.

\section{Results}

Regarding the number of meals per day of the pregnant women, only $44 \%$ of our respondents complied with the recommended number of meals per day, i.e. 3 main meals and 1-2 snacks, while $41 \%$ of all pregnant women take only 3 meals a day, presumably being the main ones. Another $14 \%$ of the respondents said they only have 2 meals a day while one of the 100 pregnant women said that they only have one meal a day.

Studies have shown that symptoms of nausea and vomiting are specific to the first trimester of pregnancy and abnormal in the third trimester; Symptoms of heartburn are normal starting by week 30 of pregnancy, and constipation are normal in weeks 10-22. Our results showed that a large number of pregnant women (44\%) report symptoms of nausea/vomiting. Regarding the sensations of retrosternal burns-pyrosis $64 \%$ of pregnant women said they have such symptoms, and especially those in 33 weeks of pregnancy, indicating that almost $2 / 3$ of pregnant women have specific symptoms of pyrosis, moreover, normal for this period. Regarding the constipation symptoms, the majority (70\%) of the pregnant women responded negatively, thus falling into the norm. To be more precise, regarding the other $30 \%$ of the pregnant women questioned to which the answer to the question was yes, it would be necessary to investigate to what extent they had such symptoms before pregnancy.

Regarding the hypothesis that the higher the biological age of the pregnant woman, the better her dietary behavior
(BMI within normal limits), after applying the formulas detailed in methodology, we observed that the average age of pregnant women in this study is 29.55 years, the initial average weight is $66.21 \mathrm{~kg}$ and the a current weight (third trimester of pregnancy) is $78.12 \mathrm{~kg}$. We note that BMI at the time of recording the pregnancy is arround the superior limit of the normal interval, suggesting that most women were overweight initially (or have been recorded by the obstetricean when they have already started to gain weight).

The average value of the score for dietary behavior and number of meals per day were further correlated and normalized, the final results being summarized in the last column of Table I. Noticeably, the dietary behavior improves by age, especially in the case of pregnant women aged over 35 years.

The risk of listeriosis and toxoplasmosis in the third trimester of pregnancy depends on the dietary behavior throughout the pregnancy. Risk assessment regarding these conditions revealed that a large percentage (68\%) of the total pregnant women consumed artisanal, raw milk cheese questioned, which could denote, on one hand, the influences of traditional Romanian consumption and, on the other hand, the distrust in the profile industry and the avoidance of processed cheeses. However, there is an increased risk of listeriosis in about two thirds of the pregnant women surveyed. The risk of toxoplasmosis is even higher since a very large percentage (91\%) of women consumed grilled beef/pork/ game, raw smoked sausages, charcuterie, figs or gizzards in pregnancy and furthermore, $24 \%$ of the pregnant women 
admitted having had contact with cats/dogs/other animals without owners during pregnancy.

The study also aimed to analyze the level of medical nutrition information and awareness of the pregnant women. The obtained results showed that a majority (72\%) of all pregnant women received dietary information from the doctor when recording the pregnancy. On the other hand, over a quarter of the pregnant women (28\%) deny having received nutritional recommendations, which denotes organizational deficiencies in the medical staff. By corroborating these data with those of eating behavior and risk of listeriosis and toxoplasmosis, as we determined that a large number of pregnant women do not meet the number of 4-5 meals a day and consume inappropriate food, we might think that, although there is some information coming from the obstetricians, more measures are needed to raise awareness among pregnant women about the risks of improper nutrition.

The pregnant women in the sample were also asked whether they follow certain dietary restrictions for health or religious reasons and the answers showed that almost one third of the pregnant women have dietary restrictions.

In order to evaluate the consumption of dietary supplements recommended before and during pregnancy, the respondents were questioned on the administration of folic acid, iron, selective multivitamins and multivitamins + minerals. Regarding folic acid, which is indicated before pregnancy and in the first trimester to reduce the fetal risk of neural tube defects and/or autism, almost one third of women $(28 \%)$ took preconceptional folic acid and $82 \%$ admitted the administration of folic acid during pregnancy, a relatively high percentage compared to the percentage of those who comply with other recommendations on proper nutrition during pregnancy. Regarding the consumption of iron, it is recommended starting from the 12th week of pregnancy. Of the pregnant women surveyed, almost two thirds (65\%) answered affirmatively regarding the administration of iron preparations during pregnancy. Twenty-seven pregnant women took iron supplements in the first trimester and did not continue after, most of them either not being informed that it is necessary to continue iron treatment, either decided to give up iron supplements due to adverse effects or the treatment was an additional financial effort. Five of the eight pregnant women who did not take iron supplements at all during pregnancy reported that they were not informed by the doctor regarding a correct diet. The results regarding other supliments during pregnancy showed that $43 \%$ of the respondents consumed selective vitamins, while $51 \%$ of the pregnant consumed complex multivitamins and multiminerals. A large percentage, $30 \%$, did not consume such supplements during pregnancy (in one form or another) and more than half of these pregnant women reported that they were not informed by the doctor, proving a lack of information in this regard. For the other pregnant women who, although informed, did not apply this recommendation, we can either conclude that they were not aware of the risks, or are afraid to consume supplements due to some disinformation, or the treatment is relatively expensive.

The research hypothesis of an existing correlation between increasing the biological age of pregnancy and a healthier lifestyle was not confirmed, as presented in Fig. 1, since the average score calculated for lifestyle is not increasing as the pregnant is older. None of our respondents stated that they would consume alcoholic beverages or drugs.

\section{Discussion}

Healty dietary behavior depends not only on medical information (14). Although most of our pregnant respondents received nutritional recommendations from the obstetrician, the compliance with these recommendations remains deficient with regard to certain unhealthy eating habits, diet restriction and supplement consumption. Our study showed that eating behavior is more appropriate with the specific dietary recommendations especially after the age of 35 , in the sense of respecting the beneficial nutritional structure of the Food Pyramid, keeping the habit of eating healthy and respecting the optimal number of meals per day. Also, lifestyle improvement was observed in the age range 25-29 years and 35-39 years. The risk of listeriosis and toxoplasmosis can be quite high due to poor hygiene of food preparation, transport and distribution, extended traditional food consumption, connections with the rural environment, inadequate food education during pregnancy, modest financial situation. Training programs for medical staff and standardized dietary evaluation are needed to ensure that pregnant women are not only informed, but also aware of the risks in case of non-compliance with the main recommendations in the field. To determine more precisely the dietary behavior of pregnant women it might be necessary to study a larger sample of patients from various backgrounds.

\section{Acknowledgements}

Not applicable.

\section{Funding}

No funding was received.

\section{Availability of data and materials}

The datasets used and/or analyzed during the current study are available from the corresponding author on reasonable request.

\section{Authors' contributions}

ACI, MMC, NT and ID collected, analyzed and interpreted the patient data regarding the eating behavior during pregnancy. REB, CAZ and OMT substantially contributed to the conception of the study and the data interpretation, drafted the manuscript and were major contributors in the writing of the manuscript. All authors read and approved the final version of the manuscript.

\section{Ethics approval and consent to participate}

Not applicable.

\section{Patient consent for publication}

Not applicable. 


\section{Competing interests}

The authors declare that they have no competing interests.

\section{References}

1. Ramakrishnan U, Grant F, Goldenberg T, Zongrone A and Martorell R: Effect of women's nutrition before and during early pregnancy on maternal and infant outcomes: A systematic review. Paediatr Perinat Epidemiol 26 (Suppl 1): 285-301, 2012.

2. American College of Obstetricians and Gynecologists: ACOG Committee opinion no. 549: Obesity in pregnancy. Obstet Gynecol 121: 213-217, 2013.

3. Haider BA and Bhutta ZA: Multiple-micronutrient supplementation for women during pregnancy. Cochrane Database Syst Rev 4: CD004905, 2017.

4. Barker DJ and Thornburg KL: The obstetric origins of health for a lifetime. Clin Obstet Gynecol 56: 511-519, 2013.

5. Kaiser L and Allen LH; American Dietetic Association: Position of the American Dietetic Association: Nutrition and lifestyle for a healthy pregnancy outcome. J Am Diet Assoc 108: 553-561, 2008.

6. Turcan N, Bohîltea R, Neacsu A, Baros A and Cîrstoiu MM: The role of anticoagulant therapy in the prevention of preeclampsia. Pharmacokinetic and pharmacodinamic mechanisms. Rev Chim 70: 1424-1428, 2019.

7. Bohîlțea R, Turcan N, Ionescu C, Toader O, Nastasia S, Neculcea D, Movileanu I, Munteanu O and Cîrstoiu M: The incidence of prematurity and associated short-term complications in a multidisciplinary emergency hospital from Romania In: Proceedings of the 5th Congress of the Romanian Society of Ultrasound in Obstetrics and Gynecology, pp105-112, 2017.
8. Bohîltea RE, Zugravu CA, Neacsu A, Navolan D, Berceanu C, Nemescu D, Bodean O, Turcan N, Baros A and Cîrstoiu MM: The prevalence of vitamin D deficiency and its obstetrical effects. A prospective study on Romanian patients. Rev Chim 70: 1228-1233, 2019.

9. Szasz F, Levai C, Navolan D, Farcas S, Andreescu N, Birsasteanu F, Mehedintu C, Ionescu CA, Bohîlțea R, Carabineanu A, et al: Weight of pregnant women and their influence on second trimester biochemical markers. REV CHIM 69: 529-532, 2018.

10. Wolff T, Witkop CT, Miller T and Syed SB; U.S. Preventive Services Task Force: Folic acid supplementation for the prevention of neural tube defects: An update of the evidence for the U.S. Preventive Services Task Force. Ann Intern Med 150: 632-639, 2009.

11. Wolf HT, Hegaard HK, Huusom LD and Pinborg AB: Multivitamin use and adverse birth outcomes in high-income countries: A systematic review and meta-analysis. Am J Obstet Gynecol 217: 404.e1-404.e30, 2017.

12. Bohiltea R, Turcan N, Cavinder CM, Ionita D, Paunica I, Andronache LF and Cirstoiu MM: Risk factors, predictive markers and prevention strategies for intrauterine fetal death. An integrative review. J Mind Med Sciences 7: 10, 2020.

13. Widen E and Siega-Riz AM: Prenatal nutrition: A practical guide for assessment and counseling. J Midwifery Womens Health 55: 540-549, 2010

14. Lucas $\mathrm{C}$, Charlton KE and Yeatman H: Nutrition advice during pregnancy: Do women receive it and can health professionals provide it? Matern Child Health J 18: 2465-2478, 2014. 